\title{
Eficácia de drogas contra Giardia muris em camundongos Mus musculus naturalmente infectados
}

\author{
Efficacy of drugs against Giardia muris in mice \\ Mus musculus naturally infected
}

\author{
Aleksandro Schafer da Silva ${ }^{1}$; Marcos Kipper da Silva ${ }^{1}$; Camila Belmonte Oliveira ${ }^{1}$; \\ Régis Adriel Zanette ${ }^{1}$; Silvia Gonzalez Monteiro ${ }^{2 *}$
}

\section{Resumo}

Este estudo visou avaliar a eficácia do metronidazol, fenbendazole e secnidazol contra Giardia muris em camundongos naturalmente infectados. Foram utilizados 40 camundongos da espécie Mus musculus divididos em quatro grupos de 10 animais cada, sendo grupo $\mathrm{A}$, grupo controle, não tratados, e grupos $\mathrm{B}, \mathrm{C}$ e D tratados com $4 \mathrm{mg} / \mathrm{ml}$ de metronidazol, fenbendazole e secnidazol, respectivamente. Para avaliar a eficácia dos medicamentos foram realizadas duas coletas de fezes uma no dia zero e outra 10 dias após tratamento. As amostras foram processadas e analisadas a partir do método de centrífugo-flutuação com sulfato de zinco. No estudo observou-se eficácia de $97,05 \%$ para metronidazol, $98,30 \%$ para fenbendazole e $100 \%$ para secnidazol no tratamento de giardiase murina. Com base nos resultados concluí-se que as drogas testadas apresentaram eficácia no tratamento de camundongos parasitados por Giardia muris. Palavras-chave: Metronidazol, fenbendazole, secnidazol, protozoário, roedores

\begin{abstract}
This study aimed to evaluate the efficacy of metronidazole, fenbendazole and secnidazole against Giardia muris in mice naturally infected. Forty mice of the species Mus musculus were divided in four groups of ten each, being group A non treated, the control group and groups B, C and D treated with $4 \mathrm{mg} / \mathrm{ml}$ of metronidazole, fenbendazole and secnidazole, respectively. Two feces collection, on day 0 and on day 10 after treatment, were done in order to evaluate the efficacy of the drugs. Samples were analyzed by the centrifugal-flotation method with zinc sulfate. Efficacy of $97,05 \%$ for metronidazole, $98,30 \%$ for fenbendazole and $100 \%$ for secnidazole were observed in the study. According to the results it was concluded that the tested drugs were effective for the treatment of mice parasitized by Giardia muris. Key words: Metronidazole, fenbendazole, secnidazole, protozoan, rodents
\end{abstract}

1 Curso de Medicina Veterinária da Universidade Federal de Santa Maria (UFSM), Santa Maria - RS, Brasil.

2 Departamento de Microbiologia e Parasitologia da UFSM. E-mail: sgmonteiro@uol.com.br.

* Autor para correspondência 
A giardíase é uma infecção comum em animais, sendo causada por um protozoário flagelado do gênero Giardia, pertencente à ordem Diplomonadida (URQUHART et al., 1987). Os animais eliminam os cistos de parasitas nas fezes após um período de prépatência de uma a duas semanas, neste período estes podem apresentar ou não sinais clínicos da enfermidade (VIGNARD-ROSEZ; ALVES; BLEICH, 2006). A contaminação por protozoários gastrintestinais em vertebrados ocorre através da ingestão de cistos presentes nas fezes, pastagens, água e alimentos (LUWWIG et al., 1999).

Dos protozoários que freqüentemente acometem os animais e o homem, Giardia sp. tem despertado grande interesse, pelo seu potencial como agente de zoonose, além de causar, em animais jovens, diarréia intermitente com comprometimento da digestão e absorção de alimentos, acarretando desidratação, perda de peso e morte. Os sinais clínicos podem ser autolimitantes em alguns pacientes e a doença grave ocorre em filhotes e em animais com doenças concomitantes ou debilitados (ROBERTS-HOMSON et al., 1976; ADAM, 1991).

Existem poucos trabalhos que enfoquem de modo sistemático e comparativo a eficácia de drogas para o tratamento de Giardia em animais. Benazet e Guillaume (1976) verificaram que metronidazole e o secnidazole, nas dosagens respectivas de $150 \mathrm{mg} / \mathrm{kg}$ e $500 \mathrm{mg} / \mathrm{kg}$ eliminam o parasito quando ministradas por dois dias consecutivos à camundongos naturalmente infectados. Conforme O'Handley et al. (1997) o fenbendazole tem sido eficaz no controle de giardíase em bezerros. Outras drogas utilizadas contra Giardia sp. como o furazolidone $(500 \mathrm{mg} / \mathrm{kg})$ e tinidazole $(200 \mathrm{mg} / \mathrm{kg})$ apresentaram eficiência de $50 \%$ e $16 \%$, respectivamente em camundongos naturalmente infectados por G. muris (CRUZ; FERRARI; SOGAYAR, 1997). Este estudo visa testar a eficácia do metronidazol, fenbendazole e secnidazol no tratamento de camundongos (Mus musculus) naturalmente infectados por Giardia muris.
O experimento foi realizado no biotério experimental do Laboratório de Parasitologia Veterinária da Universidade Federal de Santa Maria. Foram utilizados 40 camundongos da espécie $M u s$ musculus, machos, com um mês de idade, infectados naturalmente com Giardia muris, classificada de acordo com o tamanho e morfologia dos cistos encontrados nas fezes. Os roedores apresentavam uma elevada infecção por cistos do protozoário, detectada a partir de exame parasitológico de fezes. Nestes animais foram observados sinais clínicos como diarréia, pêlo seco e sem brilho, sendo que alguns animais haviam morrido devido à enfermidade.

Os camundongos foram divididos em quatro grupos, de 10 animais cada. Os roedores do Grupo A foram utilizados como grupo controle, não tratados, e os dos grupos B, C e D receberam via oral $4 \mathrm{mg} / \mathrm{ml}$ de metronidazol, fenbendazole e secnidazol, respectivamente. As drogas foram diluídas em água destilada e oferecida aos M. musculus por um período de cinco dias consecutivos. Para avaliar a eficácia do tratamento, realizaram-se duas coletas de fezes, sendo uma antes (dia zero) e outra dez dias após o início do tratamento. As amostras foram processadas a partir do método de centrífugoflutuação com sulfato de zinco (HOFFMAN, 1987). A avaliação da carga parasitária foi baseada na classificação de Pinto et al. (1994) onde se considera infecção leve 1-100 cistos/lâmina, moderada 101-300 cistos/lâmina e elevada mais de 301 cistos/lâmina.

A análise estatística dos dados foi feita através da análise de variância (ANOVA), seguida da aplicação do teste de TUKEY para comparação entre as médias, calculando-se o coeficiente de variação para verificar a precisão dos dados (SILVA; AZEVEDO, 2002). Para se calcular a eficácia dos compostos, utilizou-se a fórmula descrita por Coles et al. (1992) (Figura 1). 
$\%$ Eficácia $=\underline{\text { média grupo controle }- \text { média grupo tratado }} \times 100$ média grupo controle
No dia zero a média de cistos eliminados pelos roedores dos grupos $\mathrm{A}, \mathrm{B}, \mathrm{C}$ e D não apresentou diferença significativa $(\mathrm{p}>0,05)$. Após o tratamento, verificou-se que os medicamentos administrados aos grupos B, C e D apresentaram diferença estatística significativa entre si e com o grupo controle, sendo que os animais dos grupos $\mathrm{B}$ e $\mathrm{C}$ apresentaram uma redução significativa no número de cistos de Giardia sp. nas fezes após tratamento, porém não eliminaram o protozoário completamente (eficácia de 97,05 e $98,30 \%$ ), o que ocorreu nos roedores do grupo D, onde a droga teve eficácia de $100 \%$ (Tabela 1). As três drogas testadas foram eficazes no controle da giardíase nos camundongos infectados, com redução maior que $95 \%$ na quantidade de cistos eliminados (SPINOSA; GÓRNIAK; BERNARDI, 1999) (Tabela 1).

Tabela 1. Teste comparativo entre drogas contra Giardia muris em camundongos naturalmente infectados.

\begin{tabular}{llccc}
\hline Grupo & Droga & \multicolumn{2}{c}{ Número de cistos por lâmina } & Redução (\%) \\
\cline { 2 - 4 } & & Dia zero & Dia dez & \\
\hline A & Não tratado & $624,8^{\mathrm{a}}$ & $568,2^{\mathrm{a}}$ & $9,00^{\mathrm{a}}$ \\
B & Metronidazol & $645,2^{\mathrm{a}}$ & $19^{\mathrm{b}}$ & $97,05^{\mathrm{b}}$ \\
C & Fenbendazole & $471,4^{\mathrm{a}}$ & $08^{\mathrm{b}}$ & $98,30^{\mathrm{b}}$ \\
D & Secnidazol & $421,5^{\mathrm{a}}$ & $0,0^{\mathrm{c}}$ & $100,00^{\mathrm{c}}$ \\
\hline
\end{tabular}

Obs: Médias seguidas por letras iguais na mesma coluna não diferem estatisticamente entre si a 5\% de probabilidade do teste de TUKEY.

Cruz et al. (1997) testaram in vivo a sensibilidade de Giardia muris a quatro drogas comumente usadas no tratamento da giardíase humana. No experimento com camundongos infectados naturalmente foram utilizados o metronidazol e furazolidone $(500 \mathrm{mg} / \mathrm{kg})$, tinidazole e secnidazol $(200 \mathrm{mg} / \mathrm{kg})$. Os autores observaram uma diferença significativa na porcentagem de cura entre os roedores tratados com metronidazol $(58,3 \%)$ e o furazolidone $(50,0 \%)$, em relação ao grupo controle; já com o tinidazole (16,0\%) e o secnidazol (40,0\%) não foi constatada esta diferença. Neste estudo as drogas metronidazol e secnidazol apresentaram eficácia satisfatória e possivelmente a diferença de resultados esteja relacionada à duração do tratamento, que no estudo anterior foi de três dias e neste experimento de cinco dias, sendo que a literatura recomenda uso contínuo por sete a dez dias (SPINOSA; GÓRNIAK; BERNARDI, 1999). Semelhante a este estudo, Benazet e Guillaume (1976) observaram eficácia de $100 \%$ com a utilização de metronidazol e secnidazol quando ministrados por dois dias consecutivos em camundongos naturalmente infectados.

Barr e Bowman (1994) testaram a eficácia do fenbendazole contra Giardia sp em cães naturalmente infectados, sendo utilizada a dose de $50 \mathrm{mg} / \mathrm{kg}$, via oral e três repetições do tratamento. Os autores concluíram que a droga quando administrada por 24 horas consecutivas apresenta eficácia contra giardíase. Neste trabalho os animais do grupo $\mathrm{C}$ tiveram redução no grau de infecção após tratamento, apesar da dosagem utilizada ser de $4 \mathrm{mg} / \mathrm{ml}$, enquanto que os índices terapêuticos sugerem uma dose de $100 \mathrm{mg} / \mathrm{ml}$ no tratamento oral para animais (HOPKINS, 2005).

Escobedo et al. (2003) observaram eficácia de $79,4 \%$ e $78,1 \%$ do mebendazole e secnidazol no tratamento de giardíase em crianças, respectivamente. Outros trabalhos verificaram eficácia de $94 \%$ para o albendazole e de $98 \%$ para o metronidazol administrados à crianças de uma escola no México (ROMERO-CABELLO et al., 1995). 
Heinrich (1984) também relataram que os medicamentos metronidazol e tinidazole apresentam bons resultados no combate a Giardia lamblia em crianças com sintomatologia clínica da enfermidade.

O fenbendazole tem apresentado bons resultados no controle de protozoários do gênero Giardia em diferentes espécies de animais. Garrossino et al. (2001) observaram eficácia de $85 \%$ no tratamento de bezerros infectados naturalmente pelo parasito e Barutzki e Schmid (1999) recomendam o fenbendazole para o tratamento de giardíase em cães.

\section{Referências}

ADAM, R. D. The biology of Giardia spp. Microbiological Reviews, Washington, v.55, n.1, p.706-732, 1991.

BARR, S. C., BOWMAN, D. D. Giardiasis in dogs and cats. Compendium on Continuing Education for the Veterinary Practitioners, Montreal, v.16, n.5, p.603-614, 1994.

BARUTZKI, D.; SCHMID, K. Significance of Giardia in dogs and possibilities of control by fenbendazole. Kleintierpraxis, Hannover, v.44, n.12, p.911-918, 1999.

BENAZET, F.; GUILLAUME, L. Activité antimicrobienne et tricomonacide du secnidazole and laboratoire. Bulletin de Societé Pathologie Exotique, Paris, v.69, n.1, p.309319, 1976.

CRUZ, C. C. P.; FERRARI, L.; SOGAYAR, R. Ensaio terapêutico na infecção por Giardia muris em camundongo com metronidazole, tinidazole, secnidazole e furazolidone. Revista da Sociedade Brasileira de Medicina Tropical, Rio de Janeiro, v.30, n.3, p.223-228, 1997.

ESCOBEDO, A. A.; CANPETE, R.; GONZALEZ, M. E.; PAREJA, A.; CIMERMAN, S.; ALMIRALL, P. A randomized trial comparing mebendazole and secnidazol for the treatment of giardiasis. Annals of Tropical Medicine and Parasitology, Liverpool, v.97, n.5, p.499-504, 2003.

GAROSSINO, K. C.; RALSTON, B. J.; MCALLISTER, T. A.; MILlIGAN, D. N.; ROYAN, G.; OLSON, M. E. Individual intake and antiparasitic efficacy of free choice mineral and fenbendazole in range calves. Veterinary Parasitology, Amsterdam, v.94, n.3, p.151-162, 2001.

HEINRICH, R. Clinical manifestations and treatment of lambliasis. Therapiewoche, Karlsruhe, v.34, n.48, p.68516854, 1984.
HOFFMAN, R. P. Diagnóstico de parasitismo veterinário. Porto Alegre: Sulina, 1987.

HOPKINS, C. Peafowl medications. 2005. Disponível em: $<\mathrm{ht} \mathrm{tp://www.hopkinslivestock.com/}$ Peafowl\%20Medications.htm>. Acesso em: 20 maio 2007.

LUWWIG, K. M.; FREI, F.; ALVARES FILHO, F.; RIBEIROPAES, J. T. Correlation between sanitation condition and intestinal parasitosis in the population of Assis, state São Paulo. Revista da Sociedade Brasileira de Medicina Tropical, Rio de Janeiro, v.32, n.5, p.547-55, 1999.

O'HANDLEY, R, M.; OLSON, M. E.; MCALLISTER, T. A.; MORCK, D. W.; JELINSKI, M.; ROYAN, G.; CHENG, K. J. Efficacy of fenbendazole for treatment of giardiasis in calves. American Journal of Veterinary Research, Chicago, v.58, n.1, p.384-388, 1997.

PINTO, R.M.; VICENTE, J.J.; NORONHA,D.; GONÇALVES, L.; GOMES, D. C. Helminth parasites of conventionally maintained laboratory mice. Memórias do Instituto Oswaldo Cruz, Rio de Janeiro, v.89, n.1, p.33-40, 1994.

ROBERTS-THOMSON, J. C.; STEVENS, D. P.; MAHMOUD, A. A. F.; WARREN, K. S. Giardiasis in the mouse: an animal model. Gastroenterology, Philadelphia, v.71, n.1, p.57-61, 1976.

ROMERO-CABELLO, R.; ROBERT, L.; MUNOZ-GARCIA, R.; TANAKA, J. Random study for the assessment of safety and efficiency of albendazole and metronidazole in the treatment of giardiasis in children. Revista Latinoamericana de Microbiologia, México, v.37, n.4, p.315-323, 1995.

SILVA, F. A. S.; AZEVEDO, C. A. V. Versão do programa computacional Assistat para o sistema operacional Windows. Revista Brasileira de Produtos Agroindustriais, Campina Grande, v.4, n.1, p.71-78, 2002.

SPINOSA, H. S.; GÓRNIAK, S. L.; BERNARDI, M. M. Farmacologia aplicada à medicina veterinária. 2.ed. Rio de Janeiro: Guanabara Koogan, 1999.

URQUHART, G. M.; ARMAUR, J.; DUNCAN, J. L.; DUN, A. M.; JENNINGS, F. W. Veterinary parasitology. New York: Longman, 1987.

VIGNARD-ROSEZ, K. S. F. V.; ALVES, F. A. R.; BLEICH, I. M. Giardiase. 2006. Disponível em: <http:// www.cepav.com.br/textos/t_giardia.htm>. Acesso em: 24 maio 2006. 